\title{
ISOMORPHISMS OF SIMPLE LIE RINGS $\left({ }^{1}\right)$
}

\author{
BY \\ EUGENE A. KLOTZ
}

Introduction. It is common knowledge that all but a handful of finite dimensional simple Lie rings stem from two sources (at least if the characteristic is 0 ): they occur as the derived rings of either simple associative rings or of the set of skew elements of simple associative rings with involution [6, Chapter X]. Jacobson has discussed the isomorphisms between such Lie rings in the finite dimensional case ([4], see also Theorem 3 below). In this paper we discuss isomorphisms between simple Lie rings arising from the above two sources in one unified theory and obtain an infinite dimensional analog of Jacobson's results (subject to a finiteness condition).

The finite dimensional analogs to our results may be found in [4]. W. S. Martindale has extended results of L. K. Hua on matrix rings over a division ring to prove part (ii) of the last corollary to Theorem 4 when $\mathfrak{A}$ and $\mathfrak{B}$ are primitive rings, $\mathfrak{A}$ has characteristic $\neq 2,3$, and contains three orthogonal idempotents whose sum is the identity [8].

The first two sections are devoted to a discussion of simple involutorial algebras. In $\$ 3$ we rephrase Jacobson's classical results in terms of simple involutorial algebras. A characterization of algebraic elements which is invariant under Lie isomorphism is given in $\$ 4$. In $\S 5$ a restricted case of the main theorem is proved for particular isomorphisms of particular matrix rings. Finally, the main theorem and its corollaries are proved in $\S 6$.

Conventions. Throughout this paper we assume that the characteristic is not 2 and that all associative rings and algebras have an identity element.

1. Simple involutorial algebras. We shall employ the customary notation and definitions in dealing with involutorial algebras (i.e., algebras with involution). In particular, the definition of an involutorial algebra $(\mathfrak{A}, J)$, and the corresponding notions of ideal, subalgebra, homomorphism, center, and simple involutorial algebra are all discussed in [5]. By an involutorial ring $(\mathfrak{A}, J)$ we shall mean an involutorial algebra over the integers.

Simple involutorial rings may be characterized quite nicely. The proof of the following lemma is straightforward.

Presented to the Society, January 24, 1966; received by the editors July 29, 1966.

(1) This paper is a revised form of the author's Yale University Ph.D. dissertation. I wish to thank Professor Nathan Jacobson for his assistance in serving as my faculty adviser. This research was partially supported by NSF Grant 18916. 
LEMMA 1. ( $(\mathfrak{A}, J)$ is a simple involutorial ring iff either

(i) $\mathfrak{A}$ itself is a simple ring with involution $J$, or

(ii) $\mathfrak{A}$ is the direct sum of two ideals which are simple as rings, and $J$ is an antiisomorphism between the ideals.

Note that (ii) is equivalent to the following characterization: $\mathfrak{A}$ is the set of ordered pairs $\left(b_{1}, b_{2}\right)$ where the $b_{i}$ are from a simple ring $\mathfrak{B}$, addition is componentwise, multiplication satisfies $\left(b_{1}, b_{2}\right)\left(b_{3}, b_{4}\right)=\left(b_{1} b_{3}, b_{4} b_{2}\right)$, and $\left(b_{1}, b_{2}\right)^{J}=\left(b_{2}, b_{1}\right)$.

We shall let $\mathfrak{S}(\mathfrak{A}, J)$ denote the Lie ring of $J$-skew elements of $(\mathfrak{A}, J)$. Thus, $\mathfrak{S}(\mathfrak{A}, J)=\left\{a \in \mathfrak{A} \mid a^{J}=-a\right\}$. Similarly, $\mathfrak{S}(\mathfrak{A}, J)$ will stand for the Jordan ring of $J$-symmetric elements of $(\mathfrak{A}, J)$. We assume that the mapping $a \rightarrow 2 a$ is onto so that we have the standard $\mathfrak{A}=\mathfrak{S}(\mathfrak{A}, J) \oplus \mathfrak{S}(\mathfrak{A}, J)$.

If $(\mathfrak{A}, J)$ is a simple involutorial ring and $\mathfrak{A}$ is a simple ring then we shall distinguish between involutions of the first kind (which are the identity on the center of $\mathfrak{A})$ and involutions of the second kind (which are not). Since $\mathfrak{A}$ is simple, its center $\Phi$ will be a field, and if $J$ is of the second kind then one can easily show that $\Phi=E(\lambda)$, where $E=\mathfrak{S}(\Phi, J)$ is the center of $(\mathfrak{A}, J)$ and $\lambda \in \mathfrak{S}(\Phi, J)$.

If $(\mathfrak{A}, J)$ is a simple involutorial ring and $\mathfrak{A}$ is not simple, then by Lemma 1 $\mathfrak{A}=\mathfrak{X} \oplus \mathfrak{X}^{J}$, where $\mathfrak{X}$ is an ideal which is simple as a ring. In this case $\mathfrak{S}(\mathfrak{A}, J)$ $=\left\{x-x^{J} \mid x \in \mathfrak{X}\right\}$ and the mapping $x-x^{J} \rightarrow x$ is easily seen to be a Lie isomorphism between $\mathfrak{S}(\mathfrak{A}, J)$ and $\mathfrak{X}_{L}(=\mathfrak{X}$ considered as a Lie ring). Consequently, if we let $\mathfrak{L}^{\prime}$ denote the derived ring of $\mathfrak{L}$ (= Lie ring generated by all commutators in $\mathfrak{L}$ ), we have $\mathfrak{S}(\mathfrak{A}, J)^{\prime} \cong \mathfrak{X}^{\prime}$ under the mapping $x-x^{J} \rightarrow x, x \in \mathfrak{X}^{\prime}$. It is because of this mapping that we are able to obtain all the usual nonexceptional simple Lie rings in the form $\mathfrak{S}(\mathfrak{A}, J)^{\prime}$, with $(\mathfrak{A}, J)$ a simple involutorial ring.

I. N. Herstein has developed some very useful information on the Lie ideal structure of a simple ring $\mathfrak{A}$ and similar results have been obtained by $\mathrm{W}$. E. Baxter for Lie rings $\mathfrak{S}(\mathfrak{A}, J), \mathfrak{A}$ simple (these are all discussed in reference [3]). The following theorem, except for one necessary adjustment, is a mere restatement of the results of Herstein and Baxter in the context of involutorial rings.

THEOREM 1. Let $(\mathfrak{A}, J)$ be a simple involutorial ring and suppose that if $\mathfrak{A}$ is simple then it is more than 16 dimensional over its center, while if it is not simple then it is not commutative. Let 3 denote the center of $\mathfrak{A}$.

(i) For any Lie ideal $\mathfrak{L}$ of $\mathfrak{S}(\mathfrak{A}, J)$ either $\mathfrak{L} \supseteq \mathfrak{S}(\mathfrak{A}, J)^{\prime}$ or $\mathfrak{L} \subseteq \subseteq(\mathbb{B}, J)$.

(ii) $\subseteq(\mathfrak{A}, J)^{\prime} /\left(\mathfrak{S}(\mathfrak{A}, J)^{\prime} \cap \mathbb{3}\right)$ is a simple Lie ring.

(iii) $\mathfrak{S}(\mathfrak{A}, J)^{\prime *}$, the subring of $\mathfrak{A}$ generated by $\mathfrak{S}(\mathfrak{A}, J)^{\prime}$, is $\mathfrak{A}$ itself. (If $\mathfrak{A}$ is not simple, we must also assume here that the dimension of $(\mathfrak{A}, J)$ over its center is greater than 8.)

Proof. If $\mathfrak{A}$ is simple then (i), (ii), and (iii) are Theorems 2, 4, and 3 of [3]. If $\mathfrak{A}=\mathfrak{X} \oplus \mathfrak{X}^{J}$ is not simple then (i) and (ii) are [3, Theorem 9] and [1, Theorem 7].

We show finally that if $\mathfrak{A}=\mathfrak{X} \oplus \mathfrak{X}^{J}, \mathfrak{X}$ simple, then $\mathfrak{S}(\mathfrak{A}, J)^{\prime *} \varsubsetneqq \mathfrak{A}$ can occur only if the dimension of $\mathfrak{X}$ over its center is $\leqq 8$. Let $\mathfrak{B}=\subseteq(\mathfrak{A}, J)^{\prime *}$ and let $\pi_{1}$ be the 
projection of $\mathfrak{B}$ into $\mathfrak{X}$ given for $b=x+y^{J} \in \mathfrak{B}, x, y \in \mathfrak{X}$, by $\pi_{1}: b \rightarrow x$; similarly, let $\pi_{2}: b \rightarrow y^{J}$ be the projection of $\mathfrak{B}$ into $\mathfrak{X}^{J}$. Now Herstein has shown that $\mathfrak{X}^{\prime *}=\mathfrak{X}$ provided $\mathfrak{X}$ is not a field [3, Theorem 3] so that the $\pi_{i}$ are onto. If one of the projections, say $\pi_{2}$, is not 1-1 then there is a $b=x+y^{J} \in \mathfrak{B}$ for which $b \pi_{2}=0$, so that $b=x \in \mathfrak{X}$. Then $\mathfrak{B} \supseteq \mathfrak{B} x \mathfrak{B} \supseteq \mathfrak{X} x \mathfrak{X}=\mathfrak{X}$ since $\mathfrak{X}$ is simple; since $\mathfrak{S}(\mathfrak{A}, J)^{\prime}$ is invariant under $J$, so is $\mathfrak{B}$. Hence $\mathfrak{B} \supseteq \mathfrak{X}^{J}$ also. Thus, $\mathfrak{B} \supseteq \mathfrak{X}+\mathfrak{X}^{J}=\mathfrak{A}$ if either projection is not 1-1. If both are 1-1 then $\pi_{1}^{-1} \pi_{2}: \mathfrak{X} \rightarrow \mathfrak{X}^{J}$ is an isomorphism, and $x \pi_{1}^{-1} \pi_{2}=y^{J}$, where $x+y^{J} \in \mathfrak{B} ; y^{J}$ is unique since $\pi_{2}$ is 1-1. Since $\left(x+y^{J}\right)^{J}=y+x^{J} \in \mathfrak{B}$ we must have $y \pi_{1}^{-1} \pi_{2}=x^{J}$. Hence, if $K=\pi_{1}^{-1} \pi_{2} J$ then $K: \mathfrak{X} \rightarrow \mathfrak{X}$ is an anti-automorphism. Since $x K=y$ if $y K=x, K$ is an involution on $\mathfrak{X}$. Furthermore, if $x \in \mathfrak{X}^{\prime}$ then $x-x^{J} \in \subseteq(\mathfrak{A}, J)^{\prime} \subseteq \mathfrak{B}$ so that $x K=-x$ if $x \in \mathfrak{X}^{\prime}$, i.e., $\mathfrak{X}^{\prime} \subseteq \subseteq(\mathfrak{X}, K)$. If $\Phi$ is the center of $\mathfrak{X}$ and $\Omega$ its algebraic closure, we may easily extend $K$ to an involution of $\mathfrak{X}_{\Omega}=\mathfrak{X} \otimes_{\oplus} \Omega$ by defining $\left(\sum x_{i} \otimes \omega_{i}\right)^{K}=\sum x_{i}^{K} \otimes \omega_{i}, x_{i} \in \mathfrak{X}, \omega_{i} \in \Omega$. If $\left[\mathfrak{X}_{\Omega}: \Omega\right]$ $=n^{2}<\infty$ then $\mathfrak{X}_{\Omega}=\Omega_{n}$ is a matrix ring. Now if $\left\{e_{i}\right\}$ are matrix units then $e_{i j} \in \mathfrak{X}_{\Omega}^{\prime}$ for $i \neq j$. Hence $e_{i j}^{K}=-e_{i j}$. Hence $e_{i j}^{K}=\left(e_{i j} e_{j i}\right)^{K}=e_{j i} e_{i j}=e_{j j}$ if $i \neq j$. In order for $K$ to be 1-1 we must therefore have $n \leqq 2$ so that the dimension of $\mathfrak{A}=\mathfrak{X} \oplus \mathfrak{X}^{J}$ is $\leqq 8$.

If $\mathfrak{X}$ is infinite dimensional we use a different argument. Baxter has shown that if the dimension of $\mathfrak{X}$ over its center is more than 16 , then $\mathfrak{X}^{\prime}=\subseteq(\mathfrak{X}, K)^{\prime}$ $\oplus[\mathfrak{S}(\mathfrak{X}, K), \mathfrak{S}(\mathfrak{X}, K)]$ and also that $\subseteq(\mathfrak{X}, K)^{\prime}=\mathfrak{S}(\mathfrak{X}, K)^{\prime}$ [1, Lemma 10]. Hence if $\mathfrak{X}^{\prime} \subseteq \mathfrak{S}(\mathfrak{X}, K)^{\prime}$ we must have $[\mathfrak{S}, \mathfrak{S}]=0$ so that $0=\left[\mathfrak{S}, \mathfrak{S}^{*}\right]=[\mathfrak{S}, \mathfrak{X}]$ by (iii) for $\mathfrak{X}$ simple. Thus, $\mathfrak{S}(\mathfrak{X}, K)$ is contained in the center of $\mathfrak{X}$. But $[\mathfrak{S}, \mathfrak{S}]=[\mathfrak{S}, \subseteq$ S ] so that $\subseteq(\mathfrak{X}, K)^{\prime}=0$. But this cannot hold unless the dimension of $\mathfrak{X}$ over its center is $\leqq 16$ by [1, Lemma 3$]$.

The next theorem shows another way in which simple involutorial algebras behave like simple algebras. We shall be particularly interested in the corollary which describes the Lie subalgebra $S\left(\mathfrak{A}_{0}, J\right)$ in a form invariant under Lie isomorphism.

THEOREM 2. Let $\left(\mathfrak{A}_{0}, J\right)$ be a finite dimensional central simple involutorial subalgebra of the involutorial algebra $(\mathfrak{A}, J)$ and suppose that the center 3 of $\mathfrak{A}$ is the same as the center of $\mathfrak{A}_{0}$. If $\mathfrak{B}=\mathfrak{C}_{\mathfrak{A}}\left(\mathfrak{A}_{0}\right)$ denotes the centralizer of $\mathfrak{A}_{0}$ in $\mathfrak{A}$ then

(i) $\mathfrak{A}=\mathfrak{A}_{0} \otimes_{\mathbb{B}} \mathfrak{B}$,

(ii) $\mathfrak{E}_{\mathfrak{R}}(\mathfrak{B})=\mathfrak{A}_{0}$,

(iii) $\mathfrak{B}$ is invariant under $J$ and if $(\mathfrak{A}, J)$ is simple then so is $(\mathfrak{B}, J)$.

Proof. (i) If $\mathfrak{A}_{0}$ is simple then the theorem is well known so we assume that $\mathfrak{X}_{0}=\mathfrak{X}_{0} \oplus \mathfrak{X}_{0}^{J}$, where $\mathfrak{X}_{0}$ is simple. If $e$ is the identity of $\mathfrak{X}_{0}$ then $e, e^{J} \in \mathbb{Z}$ and $1=e+e^{J}$. Let $\mathfrak{X}=e \mathfrak{A}$ so that $\mathfrak{X}^{J}=\mathfrak{A} e^{J}=e^{J} \mathfrak{A}$. Then $\mathfrak{A}=\mathfrak{X}+\mathfrak{X}^{J}$ and $\mathfrak{X} \supseteq \mathfrak{X}_{0}$. If $x \in \mathfrak{X} \cap \mathfrak{X}^{J}$ then $x=e a=e^{J} b, a, b \in \mathfrak{A}$, so that $x=\left(e+e^{J}\right) x=0$, and $\mathfrak{A}=\mathfrak{X} \oplus \mathfrak{X}^{J}$. Note that $\mathfrak{X}$ and $\mathfrak{X}^{J}$ are ideals.

Now $3=\Psi+\Psi^{J}$, where $\Psi$ is the center of the simple ring $\mathfrak{X}_{0}$ and hence a field. Since $\mathfrak{X} \supseteq \mathfrak{X}_{0}$ and the latter is finite dimensional central simple over $\Psi$ we have 
$\mathfrak{X}=\mathfrak{X}_{0} \oplus_{\Psi} \mathfrak{\Im}$, where $\mathfrak{\Im}=\mathfrak{C}_{\mathfrak{X}}\left(\mathfrak{X}_{0}\right)$ and $\mathfrak{C}_{\mathfrak{X}}(\mathfrak{J})=\mathfrak{X}_{0}$. Similarly, $\mathfrak{X}^{J}=\mathfrak{X}_{0}^{J} \otimes_{\Psi^{J}} \mathfrak{\Im}_{1}$ and we see that $\mathfrak{\Im}^{J}=\mathfrak{C}_{\mathfrak{X}}\left(\mathfrak{X}_{0}\right)^{J}=\mathfrak{C}_{\mathfrak{X}}\left(\mathfrak{X}_{0}^{J}\right)=\mathfrak{\Im}_{1}$ so that $\mathfrak{X}^{J}=\mathfrak{X}_{0}^{J} \otimes_{\Psi^{J}} \mathfrak{\Im}^{J}$.

Clearly $\mathfrak{\Im} \oplus \mathfrak{\Im}^{J} \subseteq \mathfrak{B}=\mathfrak{V}_{\mathfrak{A}}\left(\mathfrak{A}_{0}\right)$. If $b \in \mathfrak{B}$ then $b=x_{1}+x_{2}^{J}, x_{i} \in \mathfrak{X}$, and $\left[x_{1}, \mathfrak{A}_{0}\right]=0$ implies that $\left[x_{1}, \mathfrak{X}_{0}\right]=0$ so that $x_{1} \in \mathfrak{\Im}$. Similarly $x_{2} \in \mathfrak{\Im}$, so that $b \in \mathfrak{\Im} \oplus \mathfrak{\Im}^{J}$. Thus, $\mathfrak{B}=\mathfrak{\Im} \oplus \mathfrak{\Im}^{J}$.

Now $\mathfrak{A}_{0} \mathfrak{B}=\left(\mathfrak{X}_{0} \oplus \mathfrak{X}_{0}^{J}\right)\left(\mathfrak{\Im} \oplus \mathfrak{\Im}^{J}\right)=\mathfrak{X}_{0} \mathfrak{\Im} \oplus \mathfrak{X}_{0}^{J} \mathfrak{\Im}^{J}=\mathfrak{A}$, so there is a natural homomorphism of $\mathfrak{A}_{0} \otimes \mathfrak{B} \mathfrak{B} \rightarrow \mathfrak{A}$ given by $\sum a_{i} \otimes b_{i} \rightarrow \sum a_{i} b_{i}$. To show that this is an isomorphism, suppose that $\sum a_{i} b_{i}=0, a_{i} \in \mathfrak{A}_{0}, b_{i} \in \mathfrak{B}$. Now $a_{i}=x_{1 i}+x_{2 i}^{J}, x_{j i} \in \mathfrak{X}_{0}$, and $b_{i}=y_{1 i}+y_{2 i}^{J}, y_{j i} \in \Im$. Hence $0=\sum a_{i} b_{i}=\sum\left(x_{1 i}+x_{2 i}^{J}\right) .\left(y_{1 i}+y_{2 i}^{J}\right)=\sum x_{1 i} y_{1 i}+x_{2 i}^{J} y_{2 i}^{J}$, and $\sum x_{1 i} y_{1 i}=0=\sum x_{2 i}^{J} y_{2 i}^{J}$. Therefore $\sum x_{1 i} \otimes y_{1 i}=0$ in $\mathfrak{X}_{0} \otimes_{\Psi} \Im$ and $\sum x_{2 i}^{J} \otimes y_{2 i}^{J}=0$ in $\mathfrak{X}_{0}^{J} \otimes_{\Psi^{J}} \mathfrak{\Im}^{J}$. Since each sum is therefore 0 in $\mathfrak{A}_{0} \otimes_{\mathbb{B}} \mathfrak{B}$ we must have $\sum a_{i} \otimes b_{i}$ $=\sum x_{1 i} \otimes y_{1 i}+x_{2 i}^{J} \otimes y_{2 i}^{J}=0$. Thus, the mapping has kernel zero and $\mathfrak{A} \cong \mathfrak{A}_{0} \otimes \mathbb{B} \mathfrak{B}$.

(ii) The proof that $\mathfrak{A}_{0}=\mathfrak{E}_{\mathfrak{X}}(\mathfrak{B})$ follows along the lines of the proof just given that $\mathfrak{F}_{\mathfrak{A}}\left(\mathfrak{A}_{0}\right)=\mathfrak{\Im} \oplus \mathfrak{\Im}^{J}$.

(iii) If $b \in \mathfrak{B}$ then $\left[b, \mathfrak{A}_{0}\right]^{J}=0=\left[b^{J}, \mathfrak{A}_{0}\right]$. Hence, $b^{J} \in \mathfrak{V}_{\mathfrak{A}}\left(\mathfrak{A}_{0}\right)=\mathfrak{B}$, so that $\mathfrak{B}^{J} \subseteq \mathfrak{B}$.

If $(\mathfrak{\Im}, J)$ is an ideal in $(\mathfrak{B}, J)$ then $\mathfrak{A}_{0} \mathfrak{\Im}$ is an ideal of $\mathfrak{A}$ and since $\left(\mathfrak{A}_{0} \mathfrak{\Im}\right)^{J} \subseteq \mathfrak{A}_{0} \mathfrak{\Im}^{J}$ $\subseteq \mathfrak{A}_{0} \mathfrak{F},\left(\mathfrak{A}_{0} \mathfrak{F}, J\right)$ is an ideal of $(\mathfrak{A}, J)$. Hence, either $\left(\mathfrak{A}_{0} \mathfrak{\Im}, J\right)=(0, J)$, in which case it is clear that $\mathfrak{\Im}=0$, or $\left(\mathfrak{A}_{0} \mathfrak{\Im}, J\right)=(\mathfrak{A}, J)$ and we must show that $\mathfrak{\Im}=\mathfrak{B}$. Suppose first that $\mathfrak{A}_{0}$ is not simple and use the notation of (i). If $\mathfrak{A}_{0} \mathfrak{\Im}=\mathfrak{A}$, $\mathfrak{\Im}$ an ideal of $\mathfrak{B}$, then $\mathfrak{I}=e \mathfrak{\Im}$ is an ideal of $\mathfrak{Y}$, and $\mathfrak{X}_{0} \mathfrak{\Im}=\mathfrak{X}$. Now $\mathfrak{X}_{0}$ and $\mathfrak{X}$ are central simple over $\Psi$ and $\mathfrak{X}=\mathfrak{X}_{0} \otimes_{\Psi} \mathfrak{\Im}$ so it follows easily that $\mathfrak{\Im}=\mathfrak{F}$. Hence $\mathfrak{\Im}=\mathfrak{\Im} \oplus \mathfrak{\Im}^{J}=\mathfrak{B}$ as desired. Only minor modifications of this argument are required if $\mathfrak{A}_{0}$ is simple.

COROllaRY. Let $(\mathfrak{A}, J)$ be simple with finite dimensional subalgebra $\left(\mathfrak{A}_{0}, J\right)$ which is central simple over $\Phi$. Suppose that $\left[\mathfrak{H}_{0}: \Phi\right]>16$ if $\mathfrak{A}_{0}$ is simple and $\geqq 8$ otherwise, $[\mathfrak{U}: \Phi] /\left[\mathfrak{U}_{0}: \Phi\right]>16$ (with infinity allowed), that $\subseteq(\mathfrak{H}, J)^{\prime}$ is a simple Lie algebra, and that the centers of $\mathfrak{A}$ and $\mathfrak{A}_{0}$ are the same.

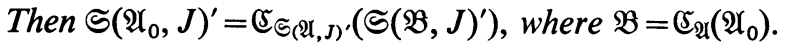

Proof. It suffices to prove

$$
\mathfrak{S}(\mathfrak{A}, J)^{\prime} \cap \mathfrak{A}_{0}=\subseteq\left(\mathfrak{A}_{0}, J\right)^{\prime} .
$$

For, since $\mathfrak{S}(\mathfrak{B}, J)^{\prime *}=\mathfrak{B}$ by Theorem 1 , and $\mathfrak{A}_{0}=\mathfrak{V}_{\mathfrak{A}}(\mathfrak{B})$ by Theorem 2 , we then would have

$$
\begin{aligned}
& \mathfrak{S}\left(\mathfrak{U}_{0}\right)^{\prime}=\mathfrak{U}_{0} \cap \mathfrak{S}(\mathfrak{H})^{\prime}=\mathfrak{V}_{\mathfrak{A}}(\mathfrak{B}) \cap \mathfrak{S}(\mathfrak{U})^{\prime}=\mathfrak{V}_{\mathbb{S}_{(\mathfrak{U})^{\prime}}}(\mathfrak{B})
\end{aligned}
$$

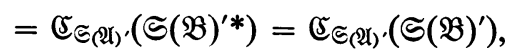

which gives the desired result.

Clearly $\mathfrak{S}\left(\mathfrak{A}_{0}\right)^{\prime} \subseteq \mathfrak{S}(\mathfrak{H})^{\prime} \cap \mathfrak{A}_{0} \subseteq \mathfrak{S}\left(\mathfrak{A}_{0}\right)$. If $\mathfrak{A}_{0}$ is simple and $J$ of the first kind then $\mathfrak{S}\left(\mathfrak{U}_{0}\right)^{\prime}=\mathfrak{S}\left(\mathfrak{A}_{0}\right)$ under our dimensionality conditions, so that (1) is immediate here [4, Theorem 9].

If $J$ is of the second kind or if $\mathfrak{A}_{0}$ is not simple we show that

$$
\mathfrak{S}\left(\mathfrak{A}_{0}\right)^{\prime}+\mathfrak{S}(\mathbb{8})=\mathfrak{S}\left(\mathfrak{A}_{0}\right) \text {, }
$$


3 the center of $\mathfrak{A}_{0}$. For then, if $x \in \mathfrak{S}(\mathfrak{A})^{\prime} \cap \mathfrak{A}_{0}$ then $x \in \mathfrak{S}\left(\mathfrak{A}_{0}\right)$ so that $x=s+z$, $s \in \mathfrak{S}\left(\mathfrak{A}_{0}\right)^{\prime}, z \in \mathfrak{S}(\mathfrak{3})$. Also, $s \in \mathfrak{S}(\mathfrak{R})^{\prime} \cap \mathfrak{A}_{0}$ so that $z \in \mathfrak{S}(\mathfrak{U})^{\prime} \cap \mathfrak{A}_{0}$. Since $\mathfrak{S}(\mathfrak{R})^{\prime}$ is assumed simple, we must have $z=0$ so $x=s \in \mathfrak{S}\left(\mathfrak{A}_{0}\right)^{\prime}$ whenever $x \in \mathfrak{S}(\mathfrak{A})^{\prime} \cap \mathfrak{A}_{0}$. This gives (1).

To show (2): If $J$ is of the second kind then $3=E(\lambda)$ with $E=\mathfrak{Y}(\Phi, J)$ and $\lambda^{J}=-\lambda$. Since $\left[\subseteq\left(\mathfrak{H}_{0}\right): E\right]=n^{2}$ and $\left[\subseteq\left(\mathfrak{H}_{0}\right)^{\prime}: E\right]=n^{2}-1[6$, p. 301], and since the simplicity of $\subseteq(\mathfrak{L})^{\prime}$ implies $\subseteq\left(\mathfrak{H}_{0}\right)^{\prime} \cap \lambda E=0$, we have $\subseteq\left(\mathfrak{A}_{0}\right)=\subseteq\left(\mathfrak{H}_{0}\right)^{\prime}+\lambda E$, $\lambda E$ being the center of $\subseteq\left(\mathfrak{A}_{0}\right)$.

If $\mathfrak{A}_{0}=\mathfrak{X} \oplus \mathfrak{X}^{J}, \mathfrak{X}$ simple with center $\Psi$ then again the simplicity of $\subseteq(\mathfrak{H})^{\prime}$ causes $\mathfrak{S}\left(\mathfrak{H}_{0}\right)^{\prime} \cap\left(\right.$ the center of $\left.\subseteq\left(\mathfrak{H}_{0}\right)\right)=0$. Now $\subseteq\left(\mathfrak{H}_{0}\right)=\left\{x-x^{J} \mid x \in \mathfrak{X}\right\}$ and $\subseteq\left(\mathfrak{H}_{0}\right)^{\prime}$ $=\left\{x-x^{J} \mid x \in \mathfrak{X}^{\prime}\right\}$, and $[\mathfrak{X}: \Psi]=n^{2}$ while $\left[\mathfrak{X}^{\prime}: \Psi\right]=n^{2}-1[4$, p. 492]. Hence $\mathfrak{X}^{\prime}+\Psi=\mathfrak{X}$. Hence $\mathfrak{S}\left(\mathfrak{H}_{0}\right)^{\prime}+\mathfrak{S}\left(\Psi \oplus \Psi^{\prime J}\right)=\mathbb{S}\left(\mathfrak{H}_{0}\right)$.

2. Central simple involutorial algebras. Let $(\mathfrak{A}, J)$ be a simple involutorial ring. The multiplication ring $\mathfrak{I}(\mathfrak{R}, J)$ of $(\mathfrak{A}, J)$ consists of the ring of endomorphisms of $\mathfrak{A}$ generated by $J$ and the left and right multiplications $a_{L}$ and $a_{R}, a \in \mathfrak{A}$. The centroid $\Gamma(\mathfrak{A}, J)$ of $(\mathfrak{A}, J)$ is the centralizer of $\mathfrak{T}(\mathfrak{U}, J)$ in the ring of endomorphisms of the additive group of $\mathfrak{A}$. Thus, $\mathfrak{T}(\mathfrak{A}, J)$ consists of the subring of $\gamma \in \Gamma(\mathfrak{A})(=$ the ordinary centroid of $\mathfrak{U}$ ) for which $\gamma J=J \gamma$. The ideals of $(\mathfrak{A}, J)$ are just the submodules of $\mathfrak{A}$ which are invariant relative to $\mathfrak{T}(\mathfrak{A}, J)$. If $(\mathfrak{A}, J)$ is simple then $\mathfrak{T}(\mathfrak{A}, J)$ is an irreducible ring of endomorphisms and $\Gamma(\mathfrak{A}, J)$ is a field by Shur's Lemma ( $\Gamma$ is commutative since our assumption of an identity forces $\mathfrak{A}^{2}=\mathfrak{A}[6$, p. 290]). One can then consider $(\mathfrak{A}, J)$ as a simple involutorial algebra over $\Gamma(\mathfrak{A}, J)$ (cf. $[6$, p. 291]).

If $(\mathfrak{A}, J)$ is a simple involutorial algebra over $\Phi$ then $(\mathfrak{A}, J)$ is central simple (i.e., $\Phi=\mathfrak{S}(3, J), 3$ the center of $\mathfrak{Q})$ iff $\Gamma(\mathfrak{A}, J)=\Phi_{R}(=$ right multiplications by elements of $\Phi)$ [6, Theorem 2]. Clearly any simple involutorial ring can be considered as a central simple involutorial algebra over its centroid.

If ( $\mathfrak{A}, J)$ is an involutorial algebra over $\Phi$ and if $\Omega$ is an extension field of $\Phi$, then we can extend $J$ to $\mathfrak{A}_{\Omega}=\mathfrak{A} \otimes_{\odot} \Omega$ by defining $\left(\sum a_{i} \otimes \omega_{i}\right)^{J}=\sum a_{i}^{J} \otimes \omega_{i}$, $a_{\mathfrak{i}} \in \mathfrak{A}, \omega_{i} \in \Omega$. $J$ is clearly an involution in $\mathfrak{A}_{\Omega}$ and $\left(\mathfrak{A}_{\Omega}, J\right)$ is thus an involutorial algebra over $\Omega$. The following two results for involutorial algebras can be established exactly as for simple nonassociative algebras without involution $[6$, Theorem 3, p. 292]:

(I) If $(\mathfrak{A}, J)$ is simple with centroid $\Gamma=\Gamma(\mathfrak{A}, J)$ then $\left(\mathfrak{U}_{\Omega}, J\right)$ is central simple for any extension field $\Omega$ of $\Gamma$.

(II) Suppose that $(\mathfrak{A}, J)$ is an algebra over $\Phi$ and that $\Lambda$ is a field with $\Gamma(\mathfrak{A}, J) \supseteq \Lambda \supseteq \Phi$. If ( $\left.\mathfrak{A} \otimes_{\Lambda} \Gamma(\mathfrak{A}, J), J\right)$ is a simple involutorial algebra over $\Lambda$ then $(\mathfrak{A}, J)$ is simple over $\Phi$ and $\Lambda=\Gamma(\mathfrak{H}, J)$.

We now turn our attention to the derived Lie ring $\subseteq(\mathfrak{A}, J)^{\prime}$ of skew elements of an involutorial ring $(\mathfrak{A}, J)$. Recall that this nonassociative ring has a multiplication ring $\mathfrak{T}\left(\subseteq(\mathfrak{L}, J)^{\prime}\right)$ and a centroid $\Gamma\left(S(\mathfrak{A}, J)^{\prime}\right)$ defined as above but without mention of $J$, and that the centroid is a field if $\subseteq(\mathfrak{A}, J)^{\prime}$ is simple. As noted, (I) and (II) hold 
for $\mathfrak{S}(\mathfrak{A}, J)^{\prime}$ if it is simple [ibid.]. If $(\mathfrak{A}, J)$ is an involutorial algebra over $\Phi$ and if $\Omega$ is an extension field of $\Phi$ we note that since we have $J$ acting as the identity on $\Omega$, then $\left.\Im_{\left(\mathfrak{A}_{\Omega}\right.}, J\right)^{\prime}=(\Im(\mathfrak{A}, J) \otimes \Omega)^{\prime}=\mathfrak{S}(\mathfrak{A}, J)^{\prime} \otimes \Omega$.

As mentioned, $\mathfrak{I}(\mathfrak{A}, J)$ is generated by $J$ and the $a_{L}$ and $a_{R}$, while $\mathfrak{I}\left(\mathfrak{S}(\mathfrak{A}, J)^{\prime}\right)$ is generated by elements ad $a=a_{R}-a_{L}, a \in \mathfrak{S}(\mathfrak{A}, J)^{\prime}$. Since $\mathfrak{T}(\mathfrak{A}, J) \supseteq \mathfrak{T}\left(\mathfrak{S}(\mathfrak{A}, J)^{\prime}\right)$ we have that $\gamma \in \Gamma(\mathfrak{A}, J)$ restricted to $\mathfrak{S}(\mathfrak{A}, J)^{\prime}$ is an element of $\Gamma\left(\mathfrak{S}(\mathfrak{A}, J)^{\prime}\right)$. Thus considered, we have $\Gamma(\mathfrak{A}, J) \subseteq \Gamma\left(\mathfrak{S}(\mathfrak{A}, J)^{\prime}\right)$.

LEMMA 2. Let $(\mathfrak{A}, J)$ be a central simple involutorial algebra which is of dimension greater than 16 if $\mathfrak{A}$ is simple, and suppose that $\mathfrak{S}(\mathfrak{A}, J)^{\prime}$ is simple.

Then $\Gamma(\mathfrak{A}, J)=\Gamma\left(\Im(\mathfrak{A}, J)^{\prime}\right)$.

Proof. For convenience let $\Gamma=\Gamma(\mathfrak{A}, J)$ and $\Phi=\Gamma\left(\Im(\mathfrak{A}, J)^{\prime}\right)$. We have noticed that $\Gamma \subseteq \Phi$ and that both are fields. By (I), ( $\left.\mathscr{U} \otimes_{\Gamma} \Phi, J\right)$ is simple. We shall show that $\mathfrak{S}\left(\mathfrak{A}_{\Phi}, J\right)^{\prime}\left(=\mathfrak{S}(\mathfrak{A}, J)^{\prime} \bigotimes_{\Gamma} \Phi\right)$ is simple so that we can apply (II) (for nonassociative algebras) and conclude that $\Gamma=\Phi$. By Theorem 1 any proper ideal of $\mathfrak{S}\left(\mathfrak{H}_{\Phi}, J\right)^{\prime}$ is contained in the center of $\mathfrak{A}_{\Phi}$. But this is $3 \otimes_{\Gamma} \Phi, 3$ the center of $\mathfrak{A}$. If $\sum c_{i} \otimes \phi \in(3 \otimes \Phi) \cap\left(S(\mathfrak{A}, J)^{\prime} \otimes \Phi\right)$ then $c_{i} \in \mathbb{Z} \cap \mathbb{S}(\mathfrak{A}, J)^{\prime}$. This latter is 0 since $\mathfrak{S}(\mathfrak{A}, J)^{\prime}$ is simple. Hence $\sum c_{i} \otimes \phi_{i}=0$ and $\mathfrak{S}\left(\mathfrak{A}_{\Phi}, J\right)^{\prime}$ is simple, as desired.

In the future we shall be concerned with Lie isomorphisms $\sigma: \mathfrak{S}(\mathfrak{A}, J)^{\prime}$ $\rightarrow \mathfrak{S}(\mathfrak{B}, K)^{\prime}$, where $\mathfrak{S}(\mathfrak{A}, J)^{\prime}$ and $\mathfrak{S}(\mathfrak{B}, K)^{\prime}$ are central simple Lie rings. Such an isomorphism induces an associative isomorphism $\sigma: \Gamma\left(\mathfrak{S}(\mathfrak{H}, J)^{\prime}\right) \rightarrow \Gamma\left(\mathfrak{S}(\mathfrak{B}, K)^{\prime}\right)$

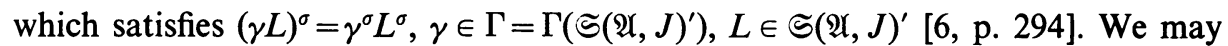
therefore define $\Gamma$ acting on $\mathfrak{S}(\mathfrak{B}, K)^{\prime}$ by $\gamma \times M=\gamma^{\sigma} M=\left(\gamma M^{\sigma^{-1}}\right)^{\sigma}, \gamma \in \Gamma$, $M \in \mathfrak{S}(\mathfrak{B}, K)^{\prime}$. It is immediate that this turns $\mathfrak{S}(\mathfrak{B}, K)^{\prime}$ into an algebra over $\Gamma$. Moreover, $\sigma$ is then a $\Gamma$-isomorphism since $(\gamma L)^{\sigma}=\gamma^{\sigma} L^{\sigma}=\gamma \times L^{\sigma}$. The centroid of $\subseteq(\mathfrak{B}, K)^{\prime}$ is clearly $\Gamma$. If $(\mathfrak{B}, K)$ is simple as in Lemma 2 then $\Gamma\left(\subseteq(\mathfrak{B}, K)^{\prime}\right)=\Gamma(\mathfrak{B}, K)$ so we can also regard $(\mathfrak{B}, K)$ as a central simple $\Gamma$-algebra, $\Gamma=\Gamma\left(\Im(\mathfrak{A}, J)^{\prime}\right)$.

If $\sigma$ is as above and if $(\mathfrak{A}, J)$ and $(\mathfrak{B}, K)$ are simple involutorial rings, we shall show that under appropriate conditions $\sigma$ can be extended to an associative isomorphism $\sigma:(\mathfrak{A}, J) \rightarrow(\mathfrak{B}, K)$. We now show that it suffices to consider the case in which $\Gamma=\Gamma\left(\mathfrak{S}(\mathfrak{A}, J)^{\prime}\right)$ is algebraically closed. For, if it is not then let $\Omega$ be its algebraic closure. By Lemma 2 and remark (I) preceding it, we have that $\mathfrak{S}\left(\mathfrak{A}_{\Omega}, J\right)^{\prime}$ and $\left(\mathfrak{A}_{\Omega}, J\right)$ are central simple over $\Omega$, where $\mathfrak{A}_{\Omega}=\mathfrak{A} \otimes_{\Gamma} \Omega$. We have just seen that we can regard $\mathfrak{S}(\mathfrak{B}, K)^{\prime}$ and $(\mathfrak{B}, K)$ as central simple over $\Gamma$. Hence, $\mathfrak{S}\left(\mathfrak{B}_{\Omega}, K\right)^{\prime}$ and $\left(\mathfrak{B}_{\Omega}, K\right)$ will be central simple over $\Omega$. Since $\mathfrak{S}\left(\mathfrak{A}_{\Omega}, J\right)^{\prime}=\mathfrak{S}(\mathfrak{A}, J)^{\prime} \otimes \Omega$, we can immediately extend $\sigma$ to an $\Omega$-isomorphism $\sigma: \mathfrak{S}\left(\mathfrak{U}_{\Omega}, J\right)^{\prime} \rightarrow \mathfrak{S}\left(\mathfrak{B}_{\Omega}, K\right)^{\prime}$ by defining $\left(\sum a_{i} \otimes \omega_{i}\right)^{\sigma}=\sum a_{i}^{\sigma} \otimes \omega_{i}, a_{i} \in \mathfrak{S}(\mathfrak{H}, J)^{\prime}, \omega_{i} \in \Omega$. Suppose then that we can extend $\sigma$ on $\mathfrak{S}\left(\mathfrak{U}_{\Omega}, J\right)^{\prime}$ to an associative isomorphism $\Sigma:\left(\mathfrak{A}_{\Omega}, J\right) \rightarrow\left(\mathfrak{B}_{\Omega}, K\right)$. Then we must have $\Sigma: \mathfrak{S}(\mathfrak{A} \otimes 1, J)^{\prime}=\mathfrak{S}(\mathfrak{A}, J)^{\prime} \otimes 1 \rightarrow \mathfrak{S}(\mathfrak{B}, K)^{\prime} \otimes 1$ and so $\Sigma: \mathfrak{S}(\mathfrak{A}, J)^{\prime *} \otimes 1$ $\rightarrow \Im(\mathfrak{B}, K)^{\prime *} \otimes 1$. For sufficiently high dimension, we may then invoke Theorem 1 to obtain that $\Sigma: \mathfrak{A} \otimes 1 \rightarrow \mathfrak{B} \otimes 1$. Consequently, we obtain by restriction an isomorphism of $(\mathfrak{A}, J) \rightarrow(\mathfrak{B}, K)$ which extends $\sigma$. 
Thus, we have seen that in considering Lie isomorphisms $\sigma: \mathfrak{S}(\mathfrak{A}, J)^{\prime} \rightarrow \mathfrak{S}(\mathfrak{B}, K)^{\prime}$ with $\mathfrak{S}(\mathfrak{A}, J)^{\prime}, \mathfrak{S}(\mathfrak{B}, K)^{\prime},(\mathfrak{A}, J)$ and $(\mathfrak{B}, K)$ central simple over $\Gamma$, it suffices to consider the case in which $\Gamma(\mathfrak{A}, J)$ is algebraically closed, provided that any assumptions made about $(\mathfrak{A}, J)$ and $(\mathfrak{B}, K)$ are preserved under passage to the algebraic closure, and that the dimension of $\mathfrak{A}$ over $\Gamma$ and $\mathfrak{B}$ over $\Gamma$ satisfies the conditions of Theorem 1.

We next consider a sufficient condition that $(\mathfrak{A}, J)$ be central simple. The proof of the following lemma is due to Jacobson.

LEMMA 3. Let $(\mathfrak{A}, J)$ be a finite dimensional involutorial algebra over $\Phi$ and suppose that

(i) $\mathfrak{S}(\mathfrak{A}, J)^{\prime}$ is central simple over $\Phi$.

(ii) $\mathfrak{A}=\mathfrak{S}(\mathfrak{A}, J)^{\prime *}$ (= the associative algebra generated by $\left.\mathfrak{S}(\mathfrak{A}, J)^{\prime}\right)$.

Then $(\mathfrak{A}, J)$ is central simple over $\Phi$.

Proof. We may assume that $\Phi$ is algebraically closed. For, consider $\mathfrak{A}_{\Omega}=\mathfrak{A} \otimes_{\Phi} \Omega$ otherwise, where $\Omega$ is the algebraic closure of $\Phi$. The hypotheses are clearly true under extension to $\Omega$, and if we can show $\left(\mathfrak{A}_{\Omega}, J\right)$ simple then $(\mathfrak{H}, J)$ will be central simple over $\Phi$ by (II) above.

If $\mathfrak{R}$ is the radical of $\mathfrak{A}$ then $\mathfrak{R}$ is the maximal nilpotent ideal of $\mathfrak{A}$ so that $\Re^{J} \subseteq \Re$. By a theorem of Mostow-Taft [9, Theorem 1] which is applicable since we can assume $\Phi$ algebraically closed, we can then write $\mathfrak{A}=\mathfrak{B}+\mathfrak{R}$, where $\mathfrak{B} \cap \mathfrak{R}=0$, the Wedderburn factor $\mathfrak{B}$ is semisimple, and $\mathfrak{B}^{J} \subseteq \mathfrak{B}$. Hence $\mathfrak{S}(\mathfrak{A}, J)=\mathfrak{S}(\mathfrak{B}, J)$ $+\mathfrak{S}(\Re, J)$, and taking derived rings we have

$$
\Im(\mathfrak{A}, J)^{\prime}=\Im(\mathfrak{B}, J)^{\prime}+\mathfrak{X}
$$

where $\mathfrak{X}=[\mathfrak{S}(\Re, J), \subseteq(\mathfrak{B}, J)]+\mathfrak{S}(\mathfrak{R}, J)^{\prime} \subseteq \Re$. If $\mathfrak{S}(\mathfrak{A}, J)^{\prime}$ is simple then since $\mathfrak{S}(\mathfrak{A}, J)^{\prime} \cap \Re$ is a nilpotent ideal in $\subseteq(\mathfrak{A}, J)^{\prime}$, this ideal must be 0 . Hence $\mathfrak{X}=0$ and $\mathfrak{S}(\mathfrak{A}, J)^{\prime}=\mathfrak{S}(\mathfrak{B}, J)^{\prime}$. But then $\mathfrak{A}=\mathfrak{S}(\mathfrak{A}, J)^{\prime *}=\mathfrak{S}(\mathfrak{B}, J)^{\prime *} \subseteq \mathfrak{B}$ so $\mathfrak{R}=0$ and $\mathfrak{A}$ is semisimple.

Suppose then that $\mathfrak{A}=\mathfrak{A}_{1} \oplus \cdots \oplus \mathfrak{A}_{n}$ is a decomposition of $\mathfrak{A}$ into ideals which are simple as rings. Since $\subseteq(\mathfrak{A}, J)^{\prime} \neq 0$ we assume that some $\mathfrak{A}_{i}$, say $\mathfrak{A}_{1}$, is not commutative. Since $\mathfrak{A}_{1}^{J}$ is also a simple ideal of $\mathfrak{A}$, either $\mathfrak{A}_{1}^{J}=\mathfrak{A}_{1}$ or $\mathfrak{A}_{1}^{J} \cap \mathfrak{A}_{1}=0$. In either case, $\mathfrak{S}\left(\mathfrak{A}_{1}+\mathfrak{U}_{1}^{J}, J\right)^{\prime}$ will be a nonzero ideal in $\mathfrak{S}(\mathfrak{A}, J)^{\prime}$. Hence it is $\mathfrak{S}(\mathfrak{A}, J)^{\prime}$, and since $\mathfrak{A}=\mathfrak{S}(\mathfrak{A}, J)^{\prime *}=\subseteq\left(\mathfrak{A}_{1}+\mathfrak{A}_{1}^{J}\right)^{\prime *} \subseteq \mathfrak{U}_{1}+\mathfrak{U}_{1}^{J}$, we see that $\mathfrak{A}=\mathfrak{A}_{1}+\mathfrak{A}_{1}^{J}$. Hence, $(\mathfrak{A}, J)$ is simple. It is central simple over $\Phi$ by Lemma 2 .

The following lemma will be used in applications of the above sufficient condition for central simplicity.

LEMMA 4. Let $\mathfrak{M}$ be a simple Lie subring of $\mathfrak{S}(\mathfrak{A}, J)^{\prime},(\mathfrak{A}, J)$ an arbitrary involutorial ring, and suppose that $\mathfrak{M}$ is the centralizer in $\mathfrak{S}(\mathfrak{A}, J)^{\prime}$ of another subring of $\mathfrak{S}(\mathfrak{A}, J)^{\prime}$.

Then $\mathfrak{M}=\mathfrak{S}\left(\mathfrak{M}^{*}, J\right)^{\prime}, \mathfrak{M}^{*}$ the enveloping associative ring of $\mathfrak{M}$. 
Proof. For convenience let $\mathfrak{L}=\mathfrak{S}(\mathfrak{A}, J)$. We are assuming that $\mathfrak{M}=\mathfrak{E}_{\mathfrak{A}}(\mathfrak{R}) \cap \mathfrak{L}^{\prime}$, where $\mathfrak{N}$ is a subring of $\mathfrak{R}$. Now by the simplicity of $\mathfrak{M}$,

$$
\mathfrak{M} \subseteq \mathfrak{S}\left(\mathfrak{M}^{*}, J\right)^{\prime}=\left(\mathfrak{M}^{*} \cap \mathfrak{L}\right)^{\prime} .
$$

Furthermore,

$$
\left(\mathfrak{M}^{*} \cap \mathfrak{Q}\right)^{\prime} \subseteq \mathfrak{M}^{*} \cap \mathfrak{Q}^{\prime}
$$

since the left-hand side is generated by elements $[x, y], x, y \in \mathfrak{M}^{*} \cap \mathfrak{L}$, and such elements $[x, y]$ are also in $\mathfrak{M}^{*} \cap \mathfrak{R}^{\prime}$. Finally, since $\mathfrak{M}^{*} \subseteq \mathfrak{C}_{\mathfrak{R}}(\mathfrak{R})$ we have

$$
\mathfrak{M}^{*} \cap \mathfrak{L}^{\prime} \subseteq \mathfrak{E}_{\mathfrak{A}}(\mathfrak{R}) \cap \mathfrak{R}^{\prime}=\mathfrak{M} .
$$

The lemma now follows from (1), (2), and (3).

3. The finite dimensional case. If $(\mathfrak{A}, J)$ is finite dimensional central simple then we can distinguish between the various types of Lie algebras $\mathfrak{S}(\mathfrak{A}, J)^{\prime}$ in the customary fashion [6, p. $302 \mathrm{ff}$.]. Thus, if $\mathfrak{A}$ is simple, $J$ is of the first kind, and $\mathfrak{A}$ is of dimension $n^{2}$ over $\Phi$, we say that $\Im(\mathfrak{A}, J)^{\prime}$ is of type

$B_{l}$ if $n=2 l+1$ and $\left[\subseteq(\mathfrak{A}, J)^{\prime}: \Phi\right]=l(2 l+1)$,

$C_{l}$ if $n=2 l$ and [S( $\left.(\mathfrak{A}, J)^{\prime}: \Phi\right]=l(2 l+1)$,

$D_{l}$ if $n=2 l$ and $\left[\Im(\mathfrak{A}, J)^{\prime}: \Phi\right]=l(2 l-1)$.

If $J$ is of the second kind then $\mathfrak{S}(\mathfrak{A}, J)^{\prime}$ is of type $A_{\mathrm{II} l}$, where $l=n-1$. If $\mathfrak{A}$ is not simple so that $\mathfrak{A}=\mathfrak{B} \oplus \mathfrak{B}^{J}$, we recall that $\mathfrak{S}(\mathfrak{A}, J)^{\prime} \cong \mathfrak{B}_{L}^{\prime}$, so that $\mathfrak{S}(\mathfrak{A}, J)^{\prime}$ is of

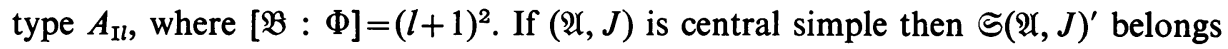
to one of the above types [4, Theorems 8 and 16]; [6, Theorems 13 and 14, p. 310].

The following theorem is essentially a rephrasing of classical results of Jacobson. We shall later obtain a generalization of relevant portions of this theorem.

TheOREM 3. Let $(\mathfrak{A}, J)$ and $(\mathfrak{B}, K)$ be finite dimensional central simple involutorial algebras of characteristic not 2. Suppose that

$l \geqq 2$ if $\mathfrak{S}(\mathfrak{U}, J)^{\prime}$ or $\mathfrak{S}(\mathfrak{B}, K)^{\prime}$ is of type $A_{\mathrm{Il}}, A_{\mathrm{IIl}}$, or $B_{l}$,

$l \geqq 3$ if $\mathfrak{S}(\mathfrak{A}, J)^{\prime}$ or $\mathfrak{S}(\mathfrak{B}, K)^{\prime}$ is of type $C_{l}$,

$l>4$ if $\mathfrak{S}(\mathfrak{A}, J)^{\prime}$ or $\mathfrak{S}(\mathfrak{B}, K)^{\prime}$ is of type $D_{l}$.

Suppose further that the characteristic does not divide $l+1$ if the type is $A_{\mathrm{Il}}$ or $A_{\mathrm{IIl}}$.

Then (i) $\mathfrak{S}(\mathfrak{A}, J)^{\prime}$ and $\mathfrak{S}(\mathfrak{B}, K)^{\prime}$ are central simple Lie algebras.

(ii) $\mathfrak{S}(\mathfrak{A}, J)^{\prime}$ and $\mathfrak{S}(\mathfrak{B}, K)^{\prime}$ are isomorphic iff $(\mathfrak{H}, J)$ and $(\mathfrak{B}, K)$ are isomorphic.

(iii) Any isomorphism of $\mathfrak{S}(\mathfrak{A}, J)^{\prime}$ onto $\mathfrak{S}(\mathfrak{B}, K)^{\prime}$ is the restriction of an isomorphism of $(\mathfrak{A}, J)$ onto $(\mathfrak{B}, K)$.

Proof. (i): $\mathfrak{S}(\mathfrak{A}, J)^{\prime}$ and $\mathfrak{S}(\mathfrak{B}, K)^{\prime}$ are simple algebras by [4, Theorem 7]; [6, Theorems 8 and 9, p. $301 \mathrm{ff}$.]. By Lemma 2 these algebras are central simple.

(ii) and (iii): As noted above we can regard an isomorphism $\sigma: \subseteq(\mathfrak{A}, J)^{\prime}$ $\rightarrow \mathfrak{S}(\mathfrak{B}, K)^{\prime}$ as an algebra isomorphism over $\Gamma=\Gamma(\mathfrak{A}, J)=\Gamma\left(\mathfrak{S}(\mathfrak{A}, J)^{\prime}\right)$, and we can consider $(\mathfrak{B}, K)$ and $\mathfrak{S}(\mathfrak{B}, K)^{\prime}$ as central simple $\Gamma$-algebras. Our conditions on $l$ assure us that there can be no isomorphism between the Lie algebras of 
different types and, at least when $\mathfrak{A}$ is simple, that (ii) and (iii) both hold [4, Theorems 1', 3', 10], [6, Theorems 11, 12, p. $306 \mathrm{ff}$.].

It only remains to consider the situation when both $\mathfrak{A}$ and $\mathfrak{B}$ are not simple. Recalling the internal characterization following Lemma 1 , we can consider $\mathfrak{A}$ as the set of ordered pairs $(x, y)$, where $x$ and $y$ are from a simple ring $\mathfrak{A}_{1}$, with appropriate addition, multiplication, and action by $J$. Similarly, $\mathfrak{B}$ is isomorphic to the set of ordered pairs $(r, s)$, where $r$ and $s$ are from a simple ring $\mathfrak{B}_{1}$. Then $\mathfrak{A}_{1}^{\prime}$ is isomorphic to $\mathfrak{S}(\mathfrak{A}, J)^{\prime}=\left\{(a,-a) \mid a \in \mathfrak{A}_{1}^{\prime}\right\}$ under $a \rightarrow(a,-a)$, and similarly $\mathfrak{S}(\mathfrak{B}, K)^{\prime}=\left\{(b,-b) \mid b \in \mathfrak{B}_{1}^{\prime}\right\}$ is isomorphic to $\mathfrak{B}_{1}^{\prime}$ under $(b,-b) \rightarrow b$. Given a Lie isomorphism $\sigma: \mathfrak{S}(\mathfrak{A}, J)^{\prime} \rightarrow \mathfrak{S}(\mathfrak{B}, K)^{\prime}$, we define a Lie isomorphism $\Sigma: \mathfrak{A}_{1}^{\prime} \rightarrow \mathfrak{B}_{1}^{\prime}$ to be the composition of the mapping we have just considered: for $a \in \mathfrak{A}_{1}^{\prime}$, if $a \rightarrow(a,-a) \rightarrow(a,-a)^{\sigma}=(b,-b) \rightarrow b$, we define $a^{\Sigma}=b$. Note that $(a,-a)^{\sigma}$ $=\left(a^{\Sigma},-a^{\Sigma}\right)$. By [4, Theorem $\left.2^{\prime}\right],[6$, Theorem 10, p. 305], $\Sigma$ can be extended to either an associative isomorphism $\Sigma$ or to the negative of an anti-isomorphism of $\mathfrak{A}_{1}$ onto $\mathfrak{B}_{1}$. In the former case, it is immediate that the mapping $\tau:(x, y) \rightarrow\left(x^{\Sigma}, y^{\Sigma}\right)$ is an associative isomorphism of $(\mathfrak{A}, J)$ onto $(\mathfrak{B}, K)$ which extends $\sigma$.

Suppose then that $\Sigma=-\alpha$, where $\alpha$ is an anti-isomorphism of $\mathfrak{A}_{1} \rightarrow \mathfrak{B}_{1}$. Define $\tau: \mathfrak{A} \rightarrow \mathfrak{B}$ by $(x, y)^{\tau}=\left(y^{\alpha}, x^{\alpha}\right)$. Then

$$
\begin{aligned}
(x, y)^{\tau}(z, w)^{\tau} & =\left(y^{\alpha}, x^{\alpha}\right)\left(w^{\alpha}, z^{\alpha}\right)=\left(y^{\alpha} w^{\alpha}, z^{\alpha} x^{\alpha}\right) \\
& =\left((w y)^{\alpha},(x z)^{\alpha}\right)=(x z, w y)^{\tau}
\end{aligned}
$$

so that $\tau$ is an associative isomorphism. Furthermore, $(x, y)^{\tau K}=\left(y^{\alpha}, x^{\alpha}\right)^{K}=\left(x^{\alpha}, y^{\alpha}\right)$ $=(y, x)^{\tau}=(x, y)^{\tau}=(x, y)^{J \tau}$ so that $\tau$ maps $(\mathfrak{A}, J)$ onto $(\mathfrak{B}, K)$. Finally, $(a,-a)^{\tau}$ $=\left(-a^{\alpha}, a^{\alpha}\right)=\left(a^{\Sigma},-a^{\Sigma}\right)=(a,-a)^{\sigma}$ so that $\tau$ is the desired extension of $\Sigma$.

We also note in regard to (ii) that one can immediately see that the restriction of any associative isomorphism between $(\mathfrak{A}, J)$ and $(\mathfrak{B}, K)$ to $\mathfrak{S}(\mathfrak{A}, J)^{\prime}$ always yields an isomorphism between $\mathfrak{S}(\mathfrak{A}, J)^{\prime}$ and $\mathfrak{S}(\mathfrak{B}, K)^{\prime}$.

4. Algebraic elements. In this section we shall develop a useful criterion that an element in $\mathfrak{S}(\mathfrak{A}, J)^{\prime}$ be algebraic.

LEMMA 5 (JACOBSON). Let $\mathfrak{A}$ be a central simple algebra over a field $\Phi$. Then an element $a \in \mathfrak{A}$ is algebraic over $\Phi$ iff ad $a=a_{L}-a_{R}$ is algebraic over $\Phi$.

Proof. If $a$ is algebraic then so are $a_{L}$ and $a_{R}$. Since $a_{L}$ and $a_{R}$ commute, every element in the algebra generated by $a_{L}$ and $a_{R}$ is algebraic. In particular, ad $a$ is algebraic.

Conversely, suppose that $a$ is transcendental. Then $1, a, a^{2}, \ldots$ are linearly independent over $\Phi$. Now $\mathfrak{A}$ is central simple so the multiplication algebra

$$
\mathfrak{T}=\left\{\sum a_{i R} b_{j L} \mid a_{i}, b_{j} \in \mathfrak{A}\right\}
$$

is isomorphic to $\mathfrak{A}_{L} \otimes \mathfrak{A}_{R}$. Since $a$ is transcendental the elements $a_{L}^{i} a_{R}^{j}, i, j=0,1$, $2, \ldots$ are linearly independent over $\Phi$. If ad $a$ were algebraic, we would have a 
relation $\left(a_{L}-a_{R}\right)^{n}=\sum_{i=0}^{n-1} \alpha_{i}\left(a_{L}-a_{R}\right)^{i}, \alpha_{i} \in \Phi$. Hence $a_{L}^{n}+(-1)^{n} a_{R}^{n}$ would be a linear combination of the elements $a_{L}^{i} a_{R}^{j}$ with $i, j \leqq n-1$, contradicting the linear independence of these elements.

LemmA 6. Let $D$ be a derivation in a nonassociative algebra $\mathfrak{A}$ over a field $\Phi$. Suppose that $\mathfrak{M}$ and $\mathfrak{N}$ are $\Phi$-subspaces of $\mathfrak{A}$ which are invariant under $D$. If $D$ is algebraic in $\mathfrak{M}$ and in $\mathfrak{N}$ then $D$ is algebraic in the $\Phi$-subspace $\mathfrak{M} \mathfrak{R}$ spanned by the products $a b, a \in \mathfrak{M}, b \in \mathfrak{R}$.

Proof. Suppose that the minimum polynomial of $D$ in $\mathfrak{M}$ is $f(\lambda)$ and in $\mathfrak{N}$ is $g(\lambda)$. We can consider $\Phi D$ as a one dimensional Lie algebra. Construct a cyclic $\Phi D$-module $[x]$ with basis $x, x D, x D^{2}, \ldots, x D^{m}$ so that the order of $x$ is $f(\lambda)$. Similarly, let $[y]$ be a cyclic $\Phi D$-module with basis $y, y D, \ldots, y D^{n}$ with $g(\lambda)$ the order of $y$. Then $[x] \otimes[y]$ is a finite dimensional $\Phi D$-module under the usual composition

$$
(x \otimes y) D=x D \otimes y+x \otimes y D
$$

for tensor products of Lie modules. Suppose that the minimum polynomial of $D$ in $[x] \otimes[y]$ is $h(\lambda)$.

Now let $a \in \mathfrak{M}, b \in \mathfrak{R}$. Then

$$
(a b) D=a D \cdot b+a \cdot b D
$$

is in $\mathfrak{M N}$ since $a D \in \mathfrak{M}$ and $b D \in \mathfrak{R}$. Hence $\mathfrak{M N}$ is invariant under $D$. Since $a f(D)=0$ and $f(\lambda)$ is the order of $x$, the cyclic $\Phi D$-module $[a]$ with basis, $a, a D, \ldots$ must be a homomorphic image of $[x]$. In like manner, the cyclic $\Phi D$-module $[b]$ must be a homomorphic image of $[y]$. Furthermore, the mapping $x D^{i} \otimes y D^{j}$ $\rightarrow a D^{i} \cdot b D^{j}$ must be a homomorphism of the module $[x] \otimes[y]$ into the module $[a][b]$ because of the operation of $D$ as given in (1) and (2). Hence $D$ is algebraic on $[a][b]$ with minimum polynomial a factor of $h(\lambda)$. In particular then, $a b h(D)=0$ for any $a \in \mathfrak{M}, b \in \mathfrak{R}$, and $D$ is algebraic on $\mathfrak{M} \mathfrak{R}$.

LEMMA 7. Let $\mathfrak{A}$ be a simple ring with involution $J$ and suppose that $\mathfrak{A}$ is more than 16 dimensional over its center $\Phi$ if $J$ is of the first kind. Then any $\Phi$-derivation $D$ of $\mathfrak{A}$ which is algebraic on $\mathfrak{S}(\mathfrak{A}, J)$ is algebraic on all of $\mathfrak{A}$.

Proof. If $J$ is of the second kind then $\mathfrak{A}=\mathfrak{S} \oplus \lambda \subseteq, \lambda \in \Phi$, and the lemma is obvious.

Baxter has shown that if the dimension is greater than 16 then every symmetric element is the sum of squares of skew-symmetric elements [1, Theorem 9]. Hence by Lemma $6, D$ is algebraic on all of $\mathfrak{A}=\mathfrak{S}(\mathfrak{A}, J) \oplus \mathfrak{S}(\mathfrak{A}, J)$.

We can now characterize algebraic elements as desired.

LemMa 8. Let $(\mathfrak{A}, J)$ be a central simple involutorial algebra over $\Phi$ of dimension greater than 16 if $\mathfrak{A}$ is simple and $J$ of the second kind.

Then an element $a \in \mathfrak{S}(\mathfrak{A}, J)^{\prime}$ is algebraic over $\Phi$ iff ad $a$ acting in $\mathfrak{S}(\mathfrak{A}, J)^{\prime}$ is algebraic over $\Phi$. 
Proof. If $\mathfrak{A}$ is simple take $D=\operatorname{ad} a$ in Lemma 7 and apply Lemma 5. If $\mathfrak{U}=\mathfrak{A}_{1} \oplus \mathfrak{A}_{1}^{J}$ is not simple, use the isomorphism between $\mathfrak{S}(\mathfrak{A}, J)^{\prime}$ and $\mathfrak{A}_{1}^{\prime}$ and apply Lemma 5 directly.

5. Matrix units. In this section we discuss matrix units and the existence of a finite dimensional simple subalgebra $\left(\mathfrak{A}_{0}, J\right)$ of a simple involutorial matrix algebra $(\mathfrak{A}, J)$. The section concludes with a lemma on the extension of Lie homomorphisms in matrix rings.

Recall that a set of elements $\left\{e_{i}\right\}, i, j=1, \ldots, n$ forms a set of matrix units in a ring $\mathfrak{A}$ if $e_{i j} e_{k l}=\delta_{j k} e_{i l}$ and $\sum_{\mathfrak{i}=1}^{n} e_{\mathfrak{i l}}=1$. If $\mathfrak{A}$ has such $n \times n$ matrix units then $\mathfrak{U}=\mathfrak{D}_{n}$ for some subring $\mathscr{D}$ of $\mathfrak{A}\left[5, \mathrm{p}\right.$. 52]. If $\mathfrak{U}=\mathfrak{B} \oplus \mathfrak{B}^{J}$ has $n \times n$ matrix units $\left\{e_{i}\right\}$ then the projections of these matrix units onto $\mathfrak{B}$ will be a set of $n \times n$ matrix units in $\mathfrak{B}$. Conversely, if $\mathfrak{B}$ has $n \times n$ matrix units $\left\{f_{i j}\right\}$ then $\left\{e_{i j}=f_{i j}+f_{j i}^{j}\right\}$ will be $n \times n$ matrix units in $\mathfrak{A}$.

In our theorem on isomorphisms of simple Lie rings of the form $\subseteq(\mathfrak{A}, J)^{\prime}$ we shall reduce the situation to Theorem 3 by assuming the existence of a finite dimensional simple subalgebra $\left(\mathfrak{A}_{0}, J\right)$ of the simple involutorial algebra $(\mathfrak{A}, J)$ with the center of $\mathfrak{A}_{0}=$ the center of $\mathfrak{A}$. We now investigate conditions for the existence of such a subalgebra $\left(\mathfrak{H}_{0}, J\right)$.

If ( $\mathfrak{A}, J$ ) is simple and possesses $n \times n$ matrix units, and if $\mathfrak{A}$ is not simple, then there is always a finite dimensional central simple subalgebra $\left(\mathfrak{A}_{0}, J\right)$ of type $A_{\mathrm{I}}$ with center $\mathfrak{A}_{0}=$ center $\mathfrak{A}$. For, $\mathfrak{A}=\mathfrak{B}_{n} \oplus \mathfrak{B}_{n}^{J}, \mathfrak{B}$ simple. If $\Phi$ is the center of $\mathfrak{B}$ let $\mathfrak{A}_{0}=\Phi_{n} \oplus \Phi_{n}^{J}$. Then $\left(\mathfrak{A}_{0}, J\right)$ is as desired since $\subseteq\left(\mathfrak{A}_{0}, J\right)^{\prime} \cong \Phi_{n}^{\prime}$. In order to consider some other cases in which such subalgebras $\left(\mathfrak{A}_{0}, J\right)$ exist, we first recall some facts which are discussed in [7].

An involution $J$ in a ring $\mathfrak{X}_{n}$ with $n \times n$ matrix units $\left\{e_{i j}\right\}$ is called canonical if for $x \in \mathfrak{X}_{n}$,

$$
x^{J}=\gamma^{-1} \bar{x}^{t} \gamma,
$$

where $t$ denotes matrix transpose, - is an involution in $\mathfrak{X}$, and $\gamma=\operatorname{diag}\left\{\gamma_{1}, \ldots, \gamma_{n}\right\}$ is a unit diagonal matrix whose entries satisfy $\bar{\gamma}_{i}=\gamma_{i}, i=1, \ldots, n$. A necessary and sufficient condition for an involution $J$ to be canonical relative to the set of matrix units $\left\{e_{i j}\right\}$ is that $e_{i i}^{J}=e_{i i}, i=1, \ldots, n$ [7, Lemma 1]. An important special type of canonical involution is the standard involution for which all the entries in $\gamma$ are the same. If $\Delta$ is a division ring then any involution in $\Delta_{n}$ is canonical relative to a suitably chosen set of matrix units [7, p. 311].

Note that if $J$ is a standard involution in the simple ring $\mathfrak{A}=\mathfrak{B}_{n}, \mathfrak{B}$ a simple ring with center $\Phi$, then $\left(\Phi_{n}, J\right)$ is a finite dimensional central simple subalgebra of $(\mathfrak{A}, J)$. For, if $\phi \in \Phi_{n}$ then $\phi^{J}=\gamma^{-1} \bar{\varphi}^{t} \gamma=\bar{\phi}^{t} \in \Phi_{n}$ since $\gamma$ is a diagonal matrix with equal diagonal entries. Thus, $\left(\Phi_{n}, J\right)$ is a subring. It is clear that $\left(\Phi_{n}, J\right)$ is simple and that $\Gamma\left(\Phi_{n}, J\right)=\Gamma(\mathfrak{U}, J)$. Combining this with our previous remarks, we see that if $(\mathfrak{A}, J)$ is a simple involutorial matrix ring and if $J$ is standard if $\mathfrak{A}$ is simple, 
then $(\mathfrak{A}, J)$ always contains a finite dimensional central simple subalgebra $\left(\mathfrak{A}_{0}, J\right)$ with center $\mathfrak{A}_{0}=$ center $\mathfrak{A}$.

We shall now let $\mathfrak{A}_{n}(n \geqq 3)$ be an arbitrary ring with $n \times n$ matrix units $\left\{f_{i j}\right\}$, and which possesses a canonical involution $J$ :

$$
x^{J}=\gamma^{-1} \bar{x}^{t} \gamma, \quad x \in \mathfrak{A}_{n} .
$$

For $i, j=1, \ldots, n$ let

$$
a\{i j\}=a f_{i j}-\left(a f_{i j}\right)^{j}=a f_{i j}-\gamma_{j}^{-1} \bar{a} \gamma_{i} f_{j i}, \quad a \in \mathfrak{A} .
$$

We note the following multiplication table:

$$
\begin{aligned}
{[a\{i j\}, b\{r s\}] } & =0 \quad \text { if } i \neq r, s \text { and } j \neq r, s . \\
{[a\{i j\}, b\{j k\}] } & =a b\{i k\}, \quad i, j, k \neq . \\
{[a\{i j\}, b\{i i\}] } & =-\left(\left(b-\gamma_{i}^{-1} \bar{b} \gamma_{i}\right) a\right)\{i j\}, \quad i \neq j . \\
{[a\{i j\}, b\{j i\}] } & =a b\{i i\}-b a\{j j\}, \quad i \neq j . \\
{[a\{i i\}, b\{i i\}] } & =\left[a_{i}, b_{i}\right]\{i i\}, \quad \text { where } x_{i}=x-\gamma_{i}^{-1} \bar{x} \gamma_{i} .
\end{aligned}
$$

From this we can see that the $a\{i j\}, i \neq j$, generate $\mathfrak{S}\left(\mathfrak{A}_{n}, J\right)^{\prime}$. For, if $\mathfrak{L}$ is the Lie ring generated by the $a\{i j\}, i \neq j$, clearly $\mathfrak{Q}$ contains everything in $\mathcal{S}\left(\mathfrak{A}_{n}, J\right)^{\prime}$ with the possible exception of elements obtained from the last equation. However, for $i, j, k \neq,[a\{i i\}-a\{j j\}, b\{i i\}-b\{k k\}]=\left[a_{i}, b_{i}\right]\{i i\}$ and is in $\mathfrak{L}$ by virtue of the next-tolast equation. Hence $\mathfrak{L} \supseteq \mathfrak{S}\left(\mathfrak{A}_{n}, J\right)^{\prime}$. Inclusion is obvious in the other direction, so we have shown that $\mathfrak{S}\left(\mathfrak{A}_{n}, J\right)^{\prime}$ is generated by the $a\{i j\}, i \neq j$.

Suppose that $\mathfrak{B}_{n}$ is another ring with $n \times n$ matrix units $\left\{e_{i j}\right\}$ and that $K$ is a canonical involution in $\mathfrak{B}_{n}$ given by

$$
y^{K}=\delta^{-1} \hat{y}^{t} \delta, \quad y \in \mathfrak{B}_{n},
$$

where $\delta=\operatorname{diag}\left\{\delta_{1}, \ldots, \delta_{n}\right\}$, and $\wedge$ is an involution in $\mathfrak{B}$. Let

$$
b(i j)=b e_{i j}-\left(b e_{i j}\right)^{J}=b e_{i j}-\delta_{j}^{-1} \hat{b} \delta_{i} e_{j i}, \quad b \in \mathfrak{B},
$$

so that the $b(i j), i \neq j$, generate $\mathfrak{S}\left(\mathfrak{B}_{n}, K\right)^{\prime}$.

LEMMA 9. Let $\left(\mathfrak{A}_{n}, J\right)$ and $\left(\mathfrak{B}_{n}, K\right)$ be arbitrary involutorial matrix rings with canonical involutions and suppose that $n \geqq 3$. Then any Lie homomorphism $\theta: \mathfrak{S}\left(\mathfrak{A}_{n}, J\right)^{\prime}$ $\rightarrow \mathfrak{S}\left(\mathfrak{B}_{n}, K\right)^{\prime}$ which satisfies $1\{i j\}^{\theta}=1(i j)$ for $i \neq j$ can be extended to an associative homomorphism

$$
T:\left(\mathfrak{A}_{n}, J\right) \rightarrow\left(\mathfrak{B}_{n}, K\right) .
$$

Proof. Let $a\{i j\}^{\theta}=\sum a_{r s}^{i j} e_{r s}$. Then for $i, j, k \neq$ we have

$$
a\{i j\}^{\theta}=\left[a\{i k\}^{\theta}, 1\{k j\}^{\theta}\right]=\left[\sum a_{r s}^{i k} e_{r s}, e_{k j}-\delta_{j}^{-1} \delta_{k} e_{k j}\right] .
$$

This shows that the matrix $a\{i j\}^{\theta}$ can have nonzero entries only in the $j$ th and $k$ th rows and columns. On the other hand,

$$
a\{i j\}^{\theta}=\left[1\{i k\}^{\theta}, a\{k j\}^{\theta}\right]=\left[e_{i k}-\delta_{k}^{-1} \delta_{i} e_{k i}, \sum a_{r s}^{k j} e_{r s}\right]
$$


so that $a\{i j\}^{\theta}$ can have nonzero entries only in the $i$ th and $k$ th rows and columns. Comparing these two computations we see that the only possible nonzero entries of $a\{i j\}^{\theta}$ are $a_{i j}^{i j}, a_{j i}^{i j}, a_{r k}^{i j}$, and $a_{k r}^{i j}, r=1, \ldots, n$. If $n \geqq 4$ we can then choose $k_{0} \neq i, j, k$ and repeat the above computations to show that the last $2 n$ possibilities are all zero. Then

$$
a\{i j\}^{\theta}=a_{i j}^{i j} e_{i j}+a_{j i}^{i j} e_{j i} \text { if } i \neq j .
$$

Equation (3) also holds if $n=3$ : It is immediate that for any $x=\left(x_{i j}\right),[x, a\{k j\}]$ has 0 in the $i i$-position, $i \neq j, k$; also, $[x, 1\{r s\}]$ has $x_{r r}-x_{s s}$ in its $r s$-position. The first fact, together with the information derived from (1) and (2), shows that $a_{k k}^{i j}=0$ for $k=1, \ldots, n$. Hence, since $a\{i j\}=[a\{i r\}, 1\{r j\}]=-[a\{r j\}, 1\{i r\}]$, the second fact shows that $a_{r j}^{i j}=0=a_{i r}^{i j}$ if $i, j, r \neq$. Since $a\{i j\}^{\theta} \in \subseteq\left(B_{n}, K\right)$ we see that $a_{j r}^{i j}=0=a_{r i}^{i j}$ if $i, j, r \neq$. Thus, (3) holds for $n=3$.

Since $a\{i j\}^{\theta}$ is in $\subseteq\left(\mathfrak{B}_{n}, K\right)^{\prime}$ for $i \neq j$, it must be of the form $b(i j)$ because of (3). We can thus write $a\{i j\}^{\theta}=a^{\tau(i j)}(i j)$. For $i, j, k \neq,[a\{i j\}, b\{j k\}]^{\theta}=\left[a\{i j\}^{\theta}, b\{j k\}^{\theta}\right]$ $=\left\{a^{\tau(i j)}(i j), b^{\tau(j k)}(j k)\right]=a^{\tau(i j)} b^{\tau(i k)}(i k)$. Hence,

$$
(a b)^{\tau(i k)}=a^{\tau(i j)} b^{\tau(i k)} \quad \text { if } i, j, k \neq .
$$

Taking $a=1$ we see that $b^{\tau(i k)}=b^{\tau(j k)}$. With $b=1$ we have $a^{\tau(i k)}=a^{\tau(i j)}$. Hence, $\tau(i j)=\tau(j k)=\tau(i k)$ is independent of $i, j, k$. We denote the common value by $\tau$. Thus, $a\{i j\}^{\theta}=a^{\tau}(i j)$ if $i \neq j . \tau$ is clearly linear and from (4), $(a b)^{\tau}=a^{\tau} b^{\tau}, 1^{\tau}=1$. Hence $\tau: \mathfrak{A} \rightarrow \mathfrak{B}$ is an associative homomorphism.

We now define a homomorphism $T: \mathfrak{A}_{n} \rightarrow \mathfrak{B}_{n}$ by $\left(a_{i j}\right)^{T}=\left(a_{i j}^{\tau}\right)$, where $\left(a_{i j}\right)$ is the matrix whose $i j$-entry is $a_{i j}$. We wish to show that $T=\theta$ on elements $a\{i j\}, i \neq j$. This would mean that $a\{i j\}^{T}=a^{\tau} e_{i j}-\left(\gamma_{j}^{-1} \bar{a} \gamma_{i}\right)^{\tau} e_{j i}$ is the same as $a\{i j\}^{\theta}=a^{\tau}(i j)$ $=a^{\tau} e_{i j}-\delta_{j}^{-1} \hat{a}^{\tau} \delta_{i} e_{j i}$, or that

$$
\left(\gamma_{j}^{-1} \bar{a} \gamma_{i}\right)^{\tau}=\delta_{j}^{-1} \hat{a}^{\tau} \delta_{i}
$$

To see that this is indeed the case we note that $a\{i j\}=-\left(\gamma_{j}^{-1} \bar{a} \gamma_{i}\right)\{j i\}$. By applying $\theta$ and equating coefficients of $e_{i j}$ we have the desired (5).

Since $T$ agrees with $\theta$ on the $a\{i j\}, i \neq j$, it will agree with $\theta$ on the Lie ring generated by these elements. As we have shown, this Lie ring is $\mathfrak{S}\left(\mathfrak{B}_{n}, K\right)^{\prime}$.

We finally show that $T:\left(\mathfrak{A}_{n}, J\right) \rightarrow\left(\mathfrak{B}_{n}, K\right)$, i.e., that $J T=T K$. But this is trivially true on $\mathfrak{S}\left(\mathfrak{A}_{n}, J\right)^{\prime}$ and hence will be true on $\mathfrak{S}\left(\mathfrak{A}_{n}, J\right)^{\prime *}$. Now for $i, j, k \neq, a\{i k\} \cdot 1\{k j\}$ $=a f_{i j}$. Thus, $\mathfrak{S}\left(\mathfrak{A}_{n}, J\right)^{\prime *}$ contains all $a f_{i j}, a \in \mathfrak{A}, i \neq j$. Clearly $\mathfrak{S}\left(\mathfrak{A}_{n}, J\right)^{\prime *}=\mathfrak{A}_{n}$, so $T:\left(\mathfrak{A}_{n}, J\right) \rightarrow\left(\mathfrak{B}_{n}, K\right)$.

6. The main theorem. We shall now prove a generalization of Theorem 3 .

THEOREM 4. Let $(\mathfrak{A}, J)$ and $(\mathfrak{B}, K)$ be simple involutorial rings of characteristic not 2. Suppose that $(\mathfrak{A}, J)$ contains a subring $\left(\mathfrak{A}_{0}, J\right)$ for which the center of $\mathfrak{A}_{0}$ is the same as the center of $\mathfrak{A}$ and that $\left(\mathfrak{A}_{0}, J\right)$ is a finite dimensional central simple involutorial algebra whose Lie algebra $\mathfrak{S}\left(\mathfrak{A}_{0}, J\right)^{\prime}$ is of type 
$A_{\mathrm{Il}}$ or $A_{\mathrm{II} l}$ with $l \geqq 2$ and the characteristic not dividing $l+1$,

$B_{l}$ with $l \geqq 2$,

$C_{l}$ with $l \geqq 3$, or

$D_{l}$ with $l>4$.

Then

(i) $\mathfrak{S}(\mathfrak{A}, J)^{\prime}$ is a central simple Lie algebra.

(ii) $\mathfrak{S}(\mathfrak{A}, J)^{\prime}$ and $\mathfrak{S}(\mathfrak{B}, K)^{\prime}$ are isomorphic iff $(\mathfrak{A}, J)$ and $(\mathfrak{B}, K)$ are isomorphic.

(iii) Any isomorphism of $\subseteq(\mathfrak{A}, J)^{\prime}$ onto $\subseteq(\mathfrak{B}, K)^{\prime}$ is the restriction of an isomorphism of $(\mathfrak{A}, J)$ onto $(\mathfrak{B}, K)$.

Proof. (i) If $\mathfrak{L} \varsubsetneqq \mathfrak{S}(\mathfrak{A}, J)^{\prime}$ is an ideal then $\mathfrak{L} \subseteq \mathbb{S} \cap \mathfrak{S}(\mathfrak{A}, J)^{\prime}$ by Theorem 1 (ii), where 3 is the center of $\mathfrak{A}$. Since 8 is also the center of $\mathfrak{A}_{0}$ we have $\mathfrak{L} \subseteq \subseteq(\mathbb{S}, J)$ $\subseteq \mathfrak{S}\left(\mathfrak{A}_{0}, J\right)$. Since $[x, \mathfrak{l}]=0$ for any $x \in \mathfrak{A}, \mathfrak{R}$ is an ideal in $\mathfrak{S}\left(\mathfrak{A}_{0}, J\right)^{\prime}$. But $\mathfrak{S}\left(\mathfrak{A}_{0}, J\right)^{\prime}$ is simple by Theorem 3 so by Theorem $1(\mathrm{i}), \mathfrak{L}=0$ and $\mathfrak{S}(\mathfrak{A}, J)^{\prime}$ is simple.

(ii) and (iii) Because of (i) we can apply Lemma 2 and obtain that $\Gamma(\mathfrak{A}, J)$ $=\Gamma\left(\mathcal{S}(\mathfrak{A}, J)^{\prime}\right)=\Gamma\left(\mathfrak{A}_{0}, J\right)=\Gamma\left(\mathfrak{S}\left(\mathfrak{A}_{0}, J\right)^{\prime}\right)$. Denote the common value by $\Gamma$. In the material following Lemma 2 we saw that if $\sigma: \mathfrak{S}(\mathfrak{A}, J)^{\prime} \rightarrow \mathfrak{S}(\mathfrak{B}, K)^{\prime}$ then $\sigma$ induces an isomorphism of the centroids so that we may consider $\mathfrak{S}(\mathfrak{B}, K)^{\prime}$ and $(\mathfrak{B}, K)$ as central simple algebras over $\Gamma$ and we may consider $\sigma$ a $\Gamma$-isomorphism. Moreover, since all our hypotheses would clearly hold after passage to the algebraic closure of $\Gamma$, we may consider $\Gamma$ as algebraically closed by the discussion preceding Lemma 3.

Let $\mathfrak{L}_{0}=\mathfrak{S}\left(\mathfrak{A}_{0}, J\right)^{\prime}$ and let $\mathfrak{B}_{0}=\mathfrak{Q}_{0}^{\sigma *}$. Our first project is to show that $\mathfrak{B}_{0}$ is a finite dimensional associative algebra over $\Gamma$. Now elements of $\mathfrak{A}_{0}$ are algebraic over $\Gamma$ so any element $L \in \mathfrak{L}_{0}$ will be algebraic over $\Gamma$. Hence ad $L$ acting in $\mathfrak{S}(\mathfrak{A}, J)^{\prime}$ will be algebraic by Lemma 8 . Hence ad $L^{\sigma}$ will be algebraic on $\Im(\mathfrak{A}, J)^{\prime \sigma}=\Im(\mathfrak{B}, K)^{\prime}$. Another application of Lemma 8 yields $L^{\sigma}$ algebraic over $\Gamma$. Let $L_{1}, \ldots, L_{n}$ be a basis for $\mathfrak{L}_{0}$ over $\Gamma$ so that $L_{1}^{\sigma}, \ldots, L_{n}^{\sigma}$ is a basis for $\mathfrak{L}_{0}^{\sigma}$ over $\Gamma$. Now $\mathfrak{B}_{0} \equiv \mathfrak{\Omega}_{0}^{\sigma *}$ is a homomorphic image of the universal enveloping algebra of $\mathfrak{R}_{0}^{\sigma}$. Hence $\mathfrak{B}_{0}$ is spanned by the monomials $\left(L_{1}^{\sigma}\right)^{r_{1}} \cdot\left(L_{2}^{\sigma}\right)^{r_{2}} \cdots\left(L_{n}^{\sigma}\right)^{r_{n}}$. Since the $L_{i}^{\sigma}$ are algebraic over $\Gamma, \mathfrak{B}_{0}$ must be finite dimensional. Note also that since $\mathfrak{L}_{0}^{\sigma K}=\mathfrak{L}_{0}^{\sigma}$, we have $\mathfrak{B}_{0}^{K} \subseteq \mathfrak{B}_{0}$, so that $\left(\mathfrak{B}_{0}, K\right)$ is a finite dimensional involutorial algebra over $\Gamma$.

By the corollary of Theorem $1, \mathfrak{L}_{0}=\mathfrak{S}\left(\mathfrak{A}_{0}, J\right)^{\prime}$ is a centralizer in $\mathfrak{S}(\mathfrak{A}, J)^{\prime}$ (if the dimensionality condition fails to hold then Theorem 3 may be applied directly to the finite dimensional $\left.\mathfrak{S}(\mathfrak{A}, J)^{\prime}\right)$. Thus, $\mathfrak{L}_{0}=\mathfrak{E}_{\mathfrak{S}_{\mathfrak{A}, J}}(\mathfrak{N})$, where $\mathfrak{N}$ is a subring of

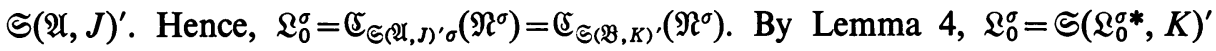
$=\mathfrak{S}\left(\mathfrak{B}_{0}, K\right)^{\prime}$. Since $\mathfrak{L}_{0}^{\sigma}$ is central simple over $\Gamma$ we can apply Lemma 3 and obtain that $\left(\mathfrak{B}_{0}, K\right)$ is simple.

We can now apply Theorem 3 to $\sigma: \mathfrak{S}\left(\mathfrak{A}_{0}, J\right)^{\prime} \rightarrow \mathfrak{Q}_{0}^{\sigma}=\mathfrak{S}\left(\mathfrak{B}_{0}, K\right)^{\prime}$. Thus, $\sigma$ can be extended to an associative isomorphism $\Sigma:\left(\mathfrak{A}_{0}, J\right) \rightarrow\left(\mathfrak{B}_{0}, K\right)$.

Now $\mathfrak{A}_{0}$ is a finite dimensional central simple algebra or the direct sum of two such, and we may assume that the base field is algebraically closed. Hence $\mathfrak{A}_{0}$ has 
$n \times n$ matrix units $\left\{f_{i i}\right\}$ and the conditions on $l$ cause $n \geqq 3$. We also note that $J$ is canonical in $\mathfrak{A}_{0}$ since $\left(\mathfrak{A}_{0}, J\right)$ is finite dimensional simple, so we may choose the matrix units to satisfy $f_{i j}^{J}=f_{i i}$. Consequently, the $\left\{f_{i i}\right\}$ will be a set of matrix units in $\mathfrak{A}$ relative to which $J$ is canonical. Applying the isomorphism $\Sigma$ we obtain matrix units $\left\{e_{i j}=f_{i j}^{\Sigma}\right\}$ in $\mathfrak{B}_{0}$ (and therefore in $\mathfrak{B}$ ). Furthermore, $e_{i \mathfrak{i}}^{K}=f_{i i}^{\Sigma K}=f_{i i}^{J \Sigma}=f_{i i}^{\Sigma}=e_{i i}$, so

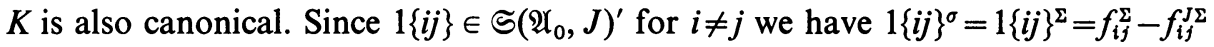
$=e_{i j}-e_{i j}^{K}=1(i j)$. By Lemma 9 we can then extend $\sigma: \mathfrak{S}(\mathfrak{A}, J)^{\prime} \rightarrow \mathfrak{S}(\mathfrak{B}, K)^{\prime}$ to an associative homomorphism of $(\mathfrak{A}, J)$ onto $(\mathfrak{B}, K)$ and this homomorphism must be an isomorphism by the simplicity of $(\mathfrak{A}, J)$.

To complete the proof, it is immediate that any isomorphism of $(\mathfrak{A}, J)$ onto $(\mathfrak{B}, K)$ yields by restriction an isomorphism of $\mathfrak{S}(\mathfrak{A}, J)^{\prime}$ onto $\mathfrak{S}(\mathfrak{B}, K)^{\prime}$.

Corollary 1. Let $(\mathfrak{A}, J)$ and $(\mathfrak{B}, K)$ satisfy the conditions of the theorem. Then any Lie isomorphism $\sigma: \mathfrak{S}(\mathfrak{A}, J) \rightarrow \mathfrak{S}(\mathfrak{B}, K)$ must be of the form $\sigma=\tau+\phi$, where $\tau:(\mathfrak{A}, J) \rightarrow(\mathfrak{B}, K)$ is an associative isomorphism and $\phi$ is an additive mapping of $(\mathfrak{A}, J)$ into the center of $(\mathfrak{B}, K)$ which vanishes on $(\mathfrak{A}, J)^{\prime}$.

Proof. Obtain $\tau$ from the theorem by restricting $\sigma$ to $\mathfrak{S}(\mathfrak{A}, J)^{\prime}$. Thus, $\sigma=\tau$ on $\cong(\mathfrak{A}, J)^{\prime}$ and it suffices to show that if $L \in \mathfrak{S}(\mathfrak{A}, J)$ then $L^{\sigma}-L^{\tau}$ lies in the center of $\subseteq(\mathfrak{B}, K)$. As shown in the proof of the theorem, we can consider $\mathfrak{B}$ as a matrix ring with elements $b(i j), i \neq j$, which generate $\subseteq(\mathfrak{B}, K)^{\prime}$. As in the proof of Lemma $9, \mathfrak{B}=\subseteq(\mathfrak{B}, K)^{\prime *} \supseteq \subseteq(\mathfrak{B}, K)$. Thus, it suffices to prove that $\left[L^{\sigma}-L^{\tau}, M^{\sigma}\right]=0$ for each $M^{\sigma} \in S(\mathfrak{B}, K)^{\prime}$. But $M^{\sigma}=M^{\tau}$ so $\left[L^{\sigma}-L^{\tau}, M^{\sigma}\right]=\left[L^{\sigma}, M^{\sigma}\right]-\left[L^{\tau}, M^{\tau}\right]$ $=[L, M]^{\sigma}-[L, M]^{\tau}=0$.

COROllary 2. Let $\mathfrak{A}$ be a central simple algebra of characteristic not 2. Suppose that $\mathfrak{A}$ contains a central simple subalgebra $\mathfrak{A}_{0}$ of finite dimension $n^{2} \geqq 9$, and that $n$ is prime to the characteristic.

(i) If $\mathfrak{B}$ is another simple ring then any Lie isomorphism of $\mathfrak{A}_{L}^{\prime}$ onto $\mathfrak{B}_{L}^{\prime}$ can be extended to either an isomorphism or the negative of an anti-isomorphism of $\mathfrak{A}$ onto $\mathfrak{B}$.

(ii) If $\mathfrak{B}$ is as in (i) then any Lie isomorphism $\sigma: \mathfrak{A}_{L} \rightarrow \mathfrak{B}_{L}$ is of the form $\sigma=\tau+\phi$, where $\tau$ is an associative isomorphism or the negative of an anti-isomorphism of $\mathfrak{A}$ onto $\mathfrak{B}$, and $\phi$ is an additive mapping into the center of $\mathfrak{B}$ which vanishes on $\mathfrak{A}_{L}^{\prime}$.

(iii) If $\mathfrak{A}_{L}^{\prime}$ is Lie isomorphic to $\subseteq(\mathfrak{B}, K)^{\prime}$, where $(\mathfrak{B}, K)$ is a simple involutorial ring, then $\mathfrak{B}=\mathfrak{B}_{0} \oplus \mathfrak{B}_{0}^{K}$ where either $\mathfrak{B}_{0}$ or $\mathfrak{B}_{0}^{K}$ is isomorphic to $\mathfrak{A}$.

Proof. (i) Take $\mathfrak{A}^{J}$ anti-isomorphic to $\mathfrak{A}, \mathfrak{B}^{K}$ anti-isomorphic to $\mathfrak{B}$ and let $\mathfrak{A}_{1}=\mathfrak{A} \oplus \mathfrak{A}^{J}, \mathfrak{B}_{1}=\mathfrak{B} \oplus \mathfrak{B}^{K}$. Then $\left(\mathfrak{A}_{1}, J\right)$ and $\left(\mathfrak{B}_{1}, K\right)$ are simple and together with $\left(\mathfrak{A}_{0} \oplus \mathfrak{A}_{0}^{J}, J\right)$, the conditions of the theorem are satisfied. The isomorphism $\sigma: \mathfrak{A}_{L}^{\prime} \rightarrow \mathfrak{B}_{L}^{\prime}$ yields an isomorphism $\sigma_{1}: \mathfrak{S}\left(\mathfrak{A}_{1}, J\right)^{\prime} \rightarrow \mathfrak{S}\left(\mathfrak{B}_{1}, K\right)^{\prime}$ given by $\left(a-a^{J}\right)^{\sigma_{1}}$ $=a^{\sigma}-a^{\sigma K}, a \in \mathfrak{A}$. By the theorem, $\sigma_{1}$ can be extended to an associative isomorphism $\tau: \mathfrak{A}_{1} \rightarrow \mathfrak{B}_{1}$. Then $\mathfrak{B}_{1}=\mathfrak{B} \oplus \mathfrak{B}^{K}$ and $\mathfrak{B}_{1}=\mathfrak{A}^{\tau} \oplus \mathfrak{U}^{J \tau}$ are two decompositions of $\mathfrak{B}_{1}$ into simple ideals. Any such decomposition is essentially unique so we must have either $\mathfrak{U}^{\mathfrak{\imath}}=\mathfrak{B}$ or $\mathfrak{U}^{\mathfrak{\imath}}=\mathfrak{B}^{K}$. If $\mathfrak{U}^{\mathfrak{\tau}}=\mathfrak{B}$ then $\tau$ is clearly the desired extension of $\sigma$. 
Suppose then that $\mathfrak{A}^{\mathfrak{\imath}}=\mathfrak{B}^{K}$. By definition $\left(a-a^{J}\right)^{\sigma_{1}}=a^{\sigma}-a^{\sigma K}$ with $a^{\sigma} \in \mathfrak{B}, a^{\sigma K} \in \mathfrak{B}^{K}$, and also $\left(a-a^{J}\right)^{\sigma_{1}}=\left(a-a^{J}\right)^{\tau}=a^{\tau}-a^{\jmath \tau}$ with $a^{\tau} \in \mathfrak{B}^{K}$. Hence $a^{\tau}=-a^{\sigma K}$, so that $a^{\sigma}=-a^{\tau K}$ and $\sigma=-\tau K$, the negative of an anti-isomorphism.

(ii) Obtain $\tau$ from (i) and proceed as in Corollary 1.

(iii) As in (i) form $\left(\mathfrak{A}_{1}, J\right)=\left(\mathfrak{A} \oplus \mathfrak{A}^{J}, J\right)$. Then $\mathfrak{S}\left(\mathfrak{A}_{1}, J\right)^{\prime} \cong \mathfrak{A}_{L}^{\prime} \cong \mathfrak{S}(\mathfrak{B}, K)^{\prime}$ and we obtain $\mathfrak{A}_{1}=\mathfrak{A} \oplus \mathfrak{U}^{J} \cong \mathfrak{B}$ from the theorem.

\section{REFERENCES}

1. W. Baxter, Lie simplicity of a special class of associative rings. II, Trans. Amer. Math. Soc. 87 (1958), 63-75.

2. I. N. Herstein, Lie and Jordan systems in simple rings with involution, Amer. J. Math. 78 (1956), 629-649.

3. (1961), 517-531.

4. N. Jacobson, Classes of restricted Lie algebras of characteristic p. I, Amer. J. Math. 63 (1941), 481-515.

5. —, Associative algebras with involution and Jordan algebras, Nederl. Akad. Wetensch. Proc. Ser. A 28 (1966), 202-212.

6. - Lie algebras, Wiley, New York, 1962.

7. N. Jacobson and C. E. Rickart, Homomorphisms of Jordan rings of self-adjoint elements, Trans. Amer. Math. Soc. 72 (1952), 310-322.

8. W. S. Martindale, III, Lie isomorphisms of primitive rings, Proc. Amer. Math. Soc. 14 (1963), 909-916.

9. E. J. Taft, Invariant Wedderburn factors, Illinois J. Math. 1 (1957), 565-573.

10. A. Weil, Algebras with involutions and the classical groups, J. Soc. Indust. Appl. Math. (1960), 589-623.

YALE UNIVERSITY,

New Haven, Connecticut 